\title{
Bringing a Cross-Disciplinary, Contextual Approach to International Service Engineering Learning
}

\section{Ms. Keilin Jahnke, University of Illinois, Urbana-Champaign}

Keilin Jahnke is a graduate student in Agricultural and Biological Engineering at the University of Illinois. She completed her Bachelor's and Master's degrees in the Industrial and Enterprise Systems Engineering department at Illinois with concentrations in sustainable international development and creativity, entrepreneurship, and innovation.

\section{Ms. Ann-Perry Witmer P.E., University of Illinois}

A teaching associate and professional civil engineer, Ann-Perry Witmer has brought to the classroom her experience working on drinking water projects with communities in the developing world. Ms. Witmer holds degrees in engineering, journalism and art history, and values the importance of developing a wellrounded understanding of the communities she serves. She has taught courses in international service design, as well as introductory engineering courses for freshmen, at the University of Illinois since 2013.

\section{Mr. Matthew Tan, University of Illinois at Urbana-Champaign}

Matthew Tan is an undergraduate student in Civil and Environmental Engineering at the University of Illinois at Urbana-Champaign. He has a passion for working on international service projects which led him to the Honduras Water Project. He has been involved since its inception in 2013 and has traveled as a mentor in 2014 and 2015.

\section{Grace Frances Witmer, University of Illinois, Urbana-Champaign}

Grace Witmer, a graduate student in Translation and Interpreting at the University of Illinois UrbanaChampaign, has ten years of experience in participating in international service projects, and served as a travelling Alumni-Mentor for the 2016 Honduras Water Project 


\title{
Bringing a Cross-Disciplinary, Contextual Approach to International Service Engineering Learning
}

\begin{abstract}
Courses in international engineering design for communities-in-need typically focus on creating, implementing, and optimizing innovative technologies that can be universally applied to the developing world. Many of the technological design assumptions are based upon the provider's values and cultural experience, and often disregard the conditions and context of the recipient community. This mindset is exacerbated by the characteristic isolation that engineering education maintains with regard to non-technical disciplines such as anthropology, community health, and social development. A year-long course at the University of Illinois at UrbanaChampaign, currently in is third year and working with its third community, breaks the isolationism of engineering thinking by combining undergraduate and graduate students in an interdisciplinary course with students in various departments to design and consult with a rural Honduran community in need of a safe drinking water supply. During the course students work closely with the community, a Honduran non-government organization, and professional advisors from various disciplines including civil engineering, water system operations, technical communications, and political governance. The course focuses on wrestling with the challenges of defining a need, evaluating alternative solutions, and devising a plan for system construction, operation, and financing. Results of the first two efforts are discussed from the perspectives of the student participants, the community recipient, the NGO, and professional partners. Feedback from course participants indicates that engineering students gain insights into the benefits of holistic engineering for domestic and international clients. Alumni of the course who continue participating as mentors report that they feel better qualified to undertake their professional responsibilities because of their course experience in defining a problem, working with a multidisciplinary team to seek an appropriate and sustainable solution, and developing the cultural awareness to view the challenge from the perspective of the target user rather than from their own viewpoint.
\end{abstract}

\section{Introduction}

Engineering education that is focused on providing service to the developing world has become a prolific trend on college campuses across the United States and beyond, spawning courses and activities that range from student organizations like Engineers Without Borders to study-abroad experiences that revolve around service actions in remote regions of the world. Academic programs often are intended to create a sense of civic engagement in the academy, yet criticism abounds that efforts are lacking substance or vision, in particular because they are not sustainable and do not foster meaningful and long-lasting change ${ }^{4}$. Researchers posit that a great number of considerations beyond practical and applied aid activities must be incorporated into service 
education, which should be a transformative experience to ensure the viability of the serviceeducation concept ${ }^{7}$. Mather recognizes that service education not only develops an awareness of need in underdeveloped societies but also develops a self-awareness, recognition of the importance that students know who they are and where they fit into the adult world. This can be particularly challenging for a generation that thrives upon rigidly defined assignments and frequent teacher or mentor feedback and control of conditions to make outcomes predictable and reliable ${ }^{4}$.

Particularly for engineering students - who are generally accustomed to course material that clearly lays out problem parameters, uses well defined methodologies, and sets rigid timelines to achieve predictable and expected outcomes - confronting ambiguity in content and execution can be deeply unsettling.

The Honduras Water Project course, now in its third year of offering at the University of Illinois at Urbana-Champaign (UIUC), addresses all the above issues by addressing engineering development contextually through use of a cross-disciplinary approach to create vision and substance; creating a transformative experience for students through self-exploration and selfdevelopment; and teaching methodology for coping with ambiguity, investing students with the confidence to think critically and nimbly while applying their knowledge to the internationalservice realm. The process is initially a painful one for students, as they adapt to a new classroom learning environment, but by the end of the year-long course, most students not only recognize but value the learned ability to work with ambiguity, adapting their understanding to the cultural understanding and value system of the recipient society rather than imposing their own expectations upon the infrastructure design. While these skills benefit the students working on international service design, they also enhance engineering skills in general by providing problem-solving techniques that are vital to the practice of engineering in the workplace ${ }^{5}$. Specifically, encouraging the students to scope the problem, gather information and assess/define solution options over time - rather than in a compressed period as would occur with a onesemester class - results in greater competency, as has been demonstrated in past research ${ }^{1}$.

The instructors of the course have participated in various extracurricular and professional international service projects. It was realized, however, that although participants of these types of projects had good intentions of helping those in need, the implemented designs often were not culturally appropriate or designed to be long-lasting. The development of the Honduras Water Project course was designed to take the good intentions of students and provide education and training to ensure that intentions were matched with good and sustainable engineering design.

Annually, the course on average has 25 participants from various disciplines, including, but not limited to: engineering; community health; Spanish; global studies; communications; accountancy, and micro and cellular biology. The students also range in level and include both 
undergraduate and graduate students. This blend of levels and majors allows for greater diversity, exposes students to perspectives different than their own, and aids in students' comprehension of the holistic nature needed for successful international engineering projects. The project also has a handful of professional advisors and alumni mentors who help the students understand their role and determine appropriate methods and actions. The professional advisors are from a variety of backgrounds, including both technical and non-technical. The alumni mentors are past students who have successfully completed at least one year of the course and volunteer their time and skills. Although the mentors and advisors are not full-time international development professionals, their added perspectives are key to helping the students discern appropriate actions and are vital to the success of the projects.

\section{The Course Objectives}

The course is founded on eight governing principles for successful projects. The lead course instructor developed these principles over time as she participated on various international service projects in Latin America and Western Africa. The governing principles are as follows:

- Vision and understanding: The project must have both vision and understanding; one without the other will lead to failure

- Community empowerment: The community must commit in writing to governing the system

- Skin in the game: The recipient community must invest in the system (financially and labor)

- Technologically appropriate: Technological design must be technologically appropriate and reviewed for quality assurance

- Culturally appropriate: Cultural considerations govern all communications as well as design approach

- Design consultants: Design team members are consultants to community, not principal stakeholders

- NGO partnership: The affiliated non-governmental organization must be an active partner in the process throughout design and construction process

- Holistic: Design must be viewed holistically in terms of both technical and cultural considerations

These governing principles serve two purposes. First, they aid the instructors in identifying potential projects with communities and NGOs. They also form the basis of the course objectives and guide the overall structure of the year as students first learn to understand the importance of these principles and then spend time applying them to the specific international engineering project. 
Since the course's inception we have partnered with a Honduran community-based organization that provides support for water distribution systems and community development. The organization, ADEC (Agua Y Desarrollo Comunitario), helps identify communities that would benefit from an improved water source and then works with them through initial data collection, implementation and construction, and long-term maintenance. ADEC is currently helping maintain at least 450 water systems in the Marcala region of Honduras. One of ADEC's hallmark characteristics is that it employs "circuit riders", individuals who routinely visit the various communities to check on the health of the systems, continue with health education programming, and train community members to better care for their systems themselves.

Each year, the students self-divide into three general categories: political, social, and technical. These teams work together to understand the cultural and political atmosphere of the community, travel to and live in the community for 10 days, and then spend the rest of the school year developing final technical designs. The Honduras Water Project has worked with ADEC to provide technical designs for a water distribution system for three different communities: Las Queseras; Fatima; and Cerro Verde. Each of these communities had approximately 200-300 residents and was looking for an improved water distribution system. The first two projects, in Las Queseras and Fatima, dealt with a groundwater source while Cerro Verde's primary source was a surface stream.

It is important to note that the students in the course do not build anything while on-site. Although many students begin the course with the expectation that they will aid in construction, the first semester exposes them to the consequences of going into a community and building a system as a charitable undertaking. Not only does this impact the amount of ownership the community feels they have of the system, the students also come to realize that they do not have the necessary construction skills, even if they come from an engineering discipline. The construction is left to both the NGO and the community. ADEC has great experience with constructing water systems in rural Honduras and employs a few master masons that are skilled at construction. The communities also often aid in construction, especially in the transport of materials, excavation, and installation of pipeline.

Overall, the course provides students with a transformative learning experience. This is necessary as each student explores international development, both to assist in identifying and resolving personal motivations and to identify the needs and expectations of the service recipient. It is the objective of the course to move students from a level of awareness, in which they acknowledge a need that must be met, through a level of attestation, in which they can observe the manifestations of that need through their own filter of understanding, to a level of assimilation, in which they can remove themselves from their own values and expectations to address the need from the perspective of the recipient ${ }^{8}$. 


\section{The Student Experience}

The Honduras Water Project is a two-semester course that immerses the student in an international service project from development through design and construction to create a sustainable, self-administered drinking water system.

The first semester focuses on developing an understanding of how to approach international projects, preliminary evaluation of options available to provide water supply, and final design of the selected approach. Additionally, presentations that introduce the importance of cultural understanding and global perspectives are given that encourage students to consider challenges aside from the obvious technical aspects. These range from the discussion of the maintenance of the system and payment for its upkeep to the property rights of the land and where the water source is located. As such, students are able to flexibly think about various issues that need to be addressed in all facets of the project. With this new mindset, the students then divide themselves into functional groups in order to address these concerns. Typically there is a technical, a social

and a political team. Students also brainstorm possible technical approaches that they can explore on the ground during their trip. They are introduced to the local NGO to start building a rapport, which will be beneficial for them to work cohesively on a solution. Professional and alumni mentors are available for them to contact if they desire advice on how to tackle these projects and move forward.

In this first semester, students are not accustomed to the teaching approach offered in this class. Rather than being provided with all the information that is available to instructors, the students are required to identify informational needs and ask for details, essentially fending for themselves. This encourages them to be independent thinkers that are able to freely consider the complex relations between technology and society that must intertwine to form a successful project. Students then break into self-defined separate functional groups that address different issues within the project—each team's first task is to determine its own responsibilities and where they fit within the overall project. As part of their preparation for their trip to Honduras, the students are required to deliver a presentation at the end of the semester that provides a detailed statement of their team's "problem," the potential solutions they considered, and the best solution they've developed and will investigate while on site. In order to prepare them for the broad approach that is needed to address these real world problems, they are also given readings to expand their perspective. The readings challenge students to examine their real motivations for choosing to take part in this project and also provide insight for how they should interact with the community. The goal of this is for students to realize why they should not be the ones who have the final say in the project but rather they are consultants to the community.

During the winter break, selected students travel to Honduras to gather sociopolitical and technical data that are crucial for the successful implementation of the project. They gain 
valuable experience as they not only collect information, but also get a real feel of the land and the culture of the community they are living in. Students are also able to interact with the leadership of the community so that they are able to hear the concerns and the needs that they wish to be addressed first-hand. Interactions between students allow this information to be passed on to the technical teams that allow them to streamline the solutions they are crafting in order to present a unique solution that caters to their needs. Additionally, they are able to test the feasibility of the engineering approaches they speculated in the previous semester, by researching which materials are available.

While in Honduras the students separate into their functional groups and begin fulfilling their deliverables identified from the previous semester. Mentors are attached to each of the groups and provide guidance on the approach the students should take. This is a crucial period where students need to use all the time available to tackle the important issues they identified from every angle. Interacting with the community is also a top priority as the students come to an understanding that they are not interacting with numbers on a page, but rather with real people and they come to the realization that the project has significant consequences on their livelihood. This is the time when the students appreciate the lessons that are given in the first semester and are better equipped to appreciate the nuances that are present when designing a solution. Meetings with the leadership (known as the 'Patronato') also allow the students to outline the expectations and deliverables of the students and the community so everyone has a clear image of what needs to be created at the close of the project. Team meetings in the evening help the students catch up on what progress has been made during the day and facilitate the sharing of information. Mentors ask the students questions, which enables them to stay focused and reflect on the impact they have on the community by their presence. This helps them assimilate into their surroundings and see the community as equals.

After the students return, their second semester focuses on preparation for construction, acquisition of materials, and implementation of system construction, funding documentation, and post-construction assessment. With the new knowledge gathered from the trip, the students are able to design the actual infrastructure that will be built by the community with ADEC's oversight. Students have ample time to conduct scale testing and experiments to determine which of their design options would be ideal for meeting the needs of the community. Meanwhile the social and political teams continue to communicate with the community and update them on the progress of the technical teams to assure them that progress is being made.

This final semester is more rigorously focused on engineering than the first part of the course, with the technical teams drafting plans for the infrastructure that will be implemented on the ground. These teams also consider how this information will be conveyed in a way that the community will be able to decipher and implement so that the product we envision is the product they receive. In order to ensure that the plans are up to standard, they are tasked to conduct 
Quality Assurance and Quality Control with a partner group. Additionally, this will be the time during which students start searching for funding. Midway through the semester, the designs are submitted to the client in order to get feedback, which will help students modify the design and address concerns regarding both construction and operation. At the end of the semester, they will have delivered the final funding, designs and construction schedule the community will follow when constructing the system. The students are actively encouraged to try as many approaches as possible without fear of failure to enable them to formulate the best solution possible.

\section{The Course Outcome}

Students in Honduras Water Project each year struggle through the first semester to define the specific need of their partner community. Especially for the less advanced engineering students, this is their first academic opportunity to assess conditions, identify client expectations, and develop a plan to solve a problem. Instructors and mentors intentionally avoid recommending specific solutions, encouraging students to instead explore their options, research alternatives, and devise a ranking methodology to determine one or two most appropriate solutions. Because the course is team-based, students also have to navigate peer personalities and opinions to complete their task of defining a need, meanwhile drawing upon the insights of non-engineering classmates to weigh technology against culture, cost, educational capabilities and operational requirements.

Throughout the process, students are required to reflect on the process as well as on their successes and struggles. Comments from students over the three years of the course are used to highlight specific learning outcomes.

- "Perhaps my view was too idealistic at the start of the course, but I am now at least aware that despite the best of intentions, many factors have to be well thought out before a large-scale project can help those in need sustainably. Furthermore, I am now aware of practical ways to account for the needs of project beneficiaries and ensure these needs are a priority at every step. I have realized that technical failures are often not as concerning as problems with societal and administrative issues that arise in such projects. For this reason, it is important for those designing the technical aspects of a project to be in close communication with people who understand the less tangible concerns in the community like its culture and internal organizational structures." -(Senior, Micro and Cellular Biology)

- "I am now more aware and considerate of why projects fail or succeed and the importance of approaching projects from different perspectives such as social, political, and technical." -(Senior, Civil Engineering) 
Lack of concrete information is a critical component of learning for many of the students, particularly the engineers. Each year, students initially assume that instructors are withholding information about the community - information such as population, demographics, elevations, distances, etc., are easily obtained in the United States, and students assume they're equally available in the developing world. In many cases, it is not until the students have traveled to the site and seen firsthand the unreliable nature of information that they understand the challenge of designing an engineered system. In one case, students saw the very name of the community change several times over the course of the first semester, then witnessed upon traveling that some residents called their town by one name and some called it by another. The lesson students eventually came to learn is that information in the developing world often is retained orally and is subject to change not only depending upon who is consulted but also upon the time of day that consultation occurs.

- "From having class discussions, it seems to me that knowing the community's culture is the first step to make appropriate plans for the project [...] The group needs to learn and understand others' cultures and lifestyles, but not being able to find much information could lead to potential problems." -(Senior, Molecular and Cellular Biology)

- "The people of the community never ceased to surprise me with their genuine care for the greater good of the community, their preparedness for the project, and their intellect. As I became more familiar with the Fatima way of life I became more aware of the living conditions as I started to visit places away from the community center and Nestor's home. Many places didn't even have pilas [water basins], let alone latrines or running water. Nestor's home seemed like a mansion compared to every other house and looks like a shack compared to the places we live in, in the United States. Going deeper into the community and seeing the houses of the guides and members of the patronato that we interacted with most often was shocking. It was shocking at first to see the faces of the community we saw go to homes that we see as barely livable, but that was the standard. These were the conditions there were born into and grew up to live in and it was normal to them. After that realization, it made me stop comparing the homes in Fatima to those in the United States, because its a different context and a different purpose." -(Junior, Engineering Physics)

Because of the variability and unreliability of accurate information that students are taught to accept, they are encouraged to evaluate alternative approaches not only for one solution but for two or even three, any of which can be adapted and modified should information about conditions change. This flies contrary to traditional engineering education, where students typically are expected to solve a discrete set of calculations to arrive at a correct solution. An example of how approach and solution can vary as conditions change could be found on this year's travel to Cerro Verde, Honduras. The community had identified a stream from which they 
currently extract water and a river at slightly higher elevation as two potential water sources for a potable water system. After evaluating likely water-quality and -quantity conditions for both the river and stream, a student team concluded the best collection design would be a streambank filtration configuration that involved digging a well beside the river. Upon arrival in the community, though, they performed several test-diggings and found that bedrock lay two feet below the ground surface and above the water table, preventing construction of a viable filtration well. Because they had assessed a series of alternatives, the team was equipped to test several other options and take measurements to design a dam and intake filter on the slower-flowing stream.

- "In addition to creating high quality designs, our teams develop innovative ideas that result from collaboration and interaction with individuals across many areas of specialty. Working with other individuals is essential because it often results in ideas that meet the needs of the community in the best way possible. This course has taught me that the human aspects are the most important areas to "get right" for a design, because without them, the system will not be successful in an international community." -(Junior, Civil Engineering)

The time spent in preparation for field investigations also allows students to draw upon each other's disciplines to understand why they should consider place, culture, politics, economics, education, health and other aspects of developing society before settling on a design solution. For many engineers, this is the first time they have been challenged in class to consider not only how a community would use a potable water system but why they would use it and what features would be desirable or inappropriate. In collaborating this way, our technical students confront a surprise realization that sustainability of a system doesn't depend solely on technical quality but on human factors as well. From this experience, students recognize that they could not quickly design a sustainable, technologically appropriate engineered solution to a developing-world need by drawing upon their book learning and anecdotal experience in the industrialized world. Instead, they need to familiarize themselves with the society they seek to serve, understand the attitudes and peculiarities of the recipient population, and craft a solution with sensitivity to recipient needs.

- This work actually challenged us to tackle a new topic, learn a new culture, and adapt a new idea of thinking. I hated the idea of not being to help physically with the development of the project. I also hated the fact that the community has the final say in everything that happens. Nothing seemed logical to me at the time and I really did not enjoy this class in the beginning, but as time went on, my views started to change and I came to like this course a lot. With the time passing, I started to really understand why we did the things we had to do and why everything seemed like it was going at such a slow pace. -(Sophomore, Civil Engineering) 
- " $[\mathrm{My}]$ expectations were very naive $[. .].[\mathrm{N}]$ ow I feel more confident moving forward because I am aware of how large the possibility of failure is. I do not think that it is impossible or that it is a bad thing, but that this field does need much work and can not be thought of as quick or easy solutions." -(Senior, Earth, Society, and Environmental Studies)

Students of the course who have since graduated and moved into the working world cite the HWP experience as having prepared them to think beyond engineering in their profession.

- "I've found that in a chemical plant setting, there are many non-engineering personnel who have a certain way of doing their work, and if you come along with a change in the process or a change in the work routine, it's often met with confrontation and contempt, even if it is an improvement in process safety. This class gave me the skill set to deal with these scenarios." -(Graduate, Chemical Engineering $)^{6}$

The field portion of the course cements the relationship between service provider and recipient by allowing for an immersive understanding of life in the developing world. Students do not simply visit the community - they embed within the community for a period of 10 days, living among the residents, experiencing the conditions endemic to the culture and society. During this period, students who travel invariably progress from a level of understanding of awareness to a level of attestation, and some students are able to attain a level of assimilation, in which they can assess recipient community residents' lives from outside of their own value system. Regardless of level of understanding, the students are able to personalize their understanding of need by establishing a relationship with the community, which creates within them an imperative to complete an engineered design effectively and sustainably.

The community itself, through project adherence to the Governing Principles, accepts empowerment as part of the relationship-building, as well. In January 2016, Cerro Verde was notably flexible and willing to work with alternative solutions, despite the fact that the community was loosely governed and not clearly cohesive. When proposed design issues failed to materialize, the students worked closely with the leading community members to explore alternative solutions. Perhaps most gratifying to instructors and mentors was witnessing students from wide-ranging backgrounds, often from families of privilege, working with people from the village to explore and resolve problems. At first, the community drove the students' attitudes: community members were always available to provide students with additional information and to hike with them wherever they needed to go in order to provide them with any information that they thought would help the students design the best system possible. Towards the end of travel, though, the trust that community members developed in the student team inspired the students to strive for perfection. In one case, when investigations failed to generate a clear solution, the 
students resisted the temptation to give up and seek recreational time, instead bringing lunches to the site so that they could work through the daylight hours, collaborating with the political leaders of the community to find a truly acceptable solution, and maintaining high spirits despite everything.

This partnership between students and residents becomes a motivational factor after the students' return, impelling them forward when they falter on design efforts in the classroom during the second semester.

- "I learned that it is extremely important for the community to accept the project as their own. If they do not [...] the implementation and upkeep of the system will be very difficult once our team leaves [...] I think it is entirely important for the project to be centralized on the community. We are providing a service to them, and in the end, we will no longer be an integral part of the upkeep." (Junior, Civil Engineering)

- " $[\mathrm{G}]$ oing to Honduras helped me create my own purpose in this project [...] It's not just a class or an assignment anymore. It's an obligation and a desire [...] We are simply helping with a small need. Before coming I thought that they really needed our help, but now I see that I needed their help more." -(Senior, Nutrition Sciences)

\section{Discussion}

The Honduras Water Project experience, first proposed in the summer of 2013, created a new concept in engineering education on the University of Illinois at Urbana-Champaign campus by incorporating technical design with social considerations such as culture, politics and health. Students from across the UIUC campus who participated in the course have brought perspectives unique to their discipline, encouraging engineers to think not only about functionality but also about appropriateness of design.

The process was not without pain and struggle - instructors have come to recognize the characteristics of the "first-semester dip" when students become discouraged by the lack of information and the sense that they'll never do anything to help a community. Often, those same students in the second semester acknowledge the importance of the early-year struggles, saying that without building an understanding of the conditions and constraints of the developing world, they never would be able to understand the needs and expectations of the community they seek to serve. Watching this transformation is inspirational, particularly for practicing engineers who understand the challenges of addressing client expectations in developing an infrastructure design. Too often students of engineering emerge from their education with the assumption that professional practice is similar to completing a homework set, with clear parameters and a straightforward resolution, free of ambiguity. Practicing engineers, on the other hand, recognize 
that most engineering tasks are rooted in ambiguity, whether it be associated with physical conditions or with socio-political influences.

\section{Conclusions}

International service design learning that includes a cross-disciplinary experience, merging engineering with social, cultural, economic and health considerations, produces a more sustainable infrastructure outcome to meet the needs of the recipient community.

Student development of an assimilative understanding with regard to international development efforts is a slow, often painful process, but it enhances the work product by creating infrastructure attuned to the needs, values and expectations of the recipient community.

Self-determination in class responsibilities, from team formation to design exploration, allows students to build knowledge, confidence and experience with engineering design. Active encouragement of attempting and failing, followed by review of causes of failure and revision for success, teaches students to test assertions, explore options, and seek out solutions without fear of academic retribution.

Personal growth and self-discovery are significant byproducts of this course, as students build confidence in themselves, learn about other societies and cultures, and integrate their knowledge into new frameworks.

Flexibility in topic, discussion, structure and partners is key to providing a meaningful classroom experience for students, while also teaching engineers to remain nimble, contextual and critical in thought as they evaluate solutions to a design problem.

Incorporation of multiple professional and peer viewpoints, in the form of professional advisors and alumni mentors, gives students diverse perspectives as well as comfortable resources to consult outside of the classroom, and demonstrates that many approaches can exist in tackling a design challenge.

\section{Bibliography}

1. Atman, C. J.; Adams, R. S.; Cardella, M. E.; Turns, J.; Mosborg, S.; Saleem, J. Engineering Design Processes: A Comparison of Students and Expert Practitioners. Journal of Engineering Education 2007, 96 (4), 359-379. 
2. Barber, B. R. Can We Teach Civic Education and Service-Learning in a World of Privatization, Inequality, and Interdependence? Journal of College and Character 2012, 13 (1).

3. Boyd, A. C. W. I Want You To Hold My Hand--Right Now! Journal of College and Character 2012, 13 (1).

4. Butin, D. W. Rethinking the "Apprenticeship of Liberty": The Case for Academic Programs in Community Engagement in Higher Education. Journal of College and Character 2012, 13 (1).

5. Johanssen, D. H.; Johri, A.; Olds, B. M. Engineers as Problem Solvers. In Cambridge Handbook of Engineering Education Research; Cambridge University Press: Cambridge, England, 2014; pp 103-118.

6. Holistic Learning in Honduras: Students Join with Other Undergrads to Design Water Systems for Rural Communities. Mass Transfer 2015, 8-10.

7. Mather, P. C.; Karbley, M.; Yamamoto, M. Identity Matters in a Short-Term, International ServiceLearning Program. Journal of College and Character 2012, 13 (1).

8. Witmer, A. (2015). The Influence of Non-Technical Factors on Sustainability of Engineered Infrastructure in Developing Communities. Unpublished. 